\title{
Attenuation of ischaemia reperfusion injury by human thioredoxin
}

Tatsuo Fukuse, Toshiki Hirata, Hiroyasu Yokomise, Seiki Hasegawa, Kenji Inui, Akira Mitsui, Tadashi Hirakawa, Shigeki Hitomi, Junji Yodoi, Hiromi Wada

\begin{abstract}
Background - Active oxygen species are thought to play a part in ischaemia reperfusion injury. The ability of a novel agent, human thioredoxin (hTRX), to attenuate lung damage has been examined in a rat model of ischaemia reperfusion injury.

Methods - Twenty eight animals were studied. At thoracotomy the left main bronchus and the left main pulmonary artery were clamped for 75 minutes and the lung was then reperfused for 20 minutes. Phosphate buffered saline was administered intravenously to nine control animals and hTRX (30 $\mu$ g/g body weight) was given intravenously to another group of nine animals. Two experiments were carried out. The first (Exp 1) was a time matched pair experiment (five treated, five controls), and the second (Exp 2) was performed under controlled conditions (four treated, four controls; temperature $25^{\circ} \mathrm{C}$, humidity $65 \%$ ). In another 10 nonischaemic rats and those in Exp 1 biochemical measurements of lipid peroxide, superoxide dismutase, and glutathione peroxide levels were performed.
\end{abstract}

Department of

Thoracic Surgery,

Chest Disease

Research Institute,

Kyoto University,

Shogoin, Sakyo-ku,

Kyoto 606, Japan

T Fukuse

T Hirata

$\mathrm{H}$ Yokomise

$S$ Hasegawa

$\mathrm{K}$ Inui

S Hitomi

H Wada

Central Research

Laboratory,

Ajinomoto Co Ltd,

Kawasaki, Japan

A Mitsui

T Hirakawa

Virus Research

Institute, Kyoto

University,

Yoshida-Konoe,

Sakyo-ku, Kyoto,

Japan

J Yodoi

Reprint requests to: Dr H Wada.

Received 7 April 1993

Returned to authors

Returned to auth

18 August 1993
Revised version received

Revised version received
16 June 1994
Accepted for publication

13 December 1994
Results - In both experiments rats perfused with $h T R X$ survived longer than controls. In Exp 1 the arterial oxygen tension $\left(\mathrm{PaO}_{2}\right)$ on air in the hTRX group was higher at 20 minutes than at one minute after reperfusion. In Exp $2 \mathrm{PaO}_{2}$ at 20 minutes was higher in the hTRX group than in the controls. Lipid peroxide, superoxide dismutase, and glutathione peroxide levels in the control group were higher than in the hTRX group and in the non-ischaemic groups. Histological examination showed less thickening and oedema of the alveolar walls in the hTRX group than in controls. Conclusions - These results suggest that hTRX is effective as a radical scavenger and can limit the extent of ischaemia reperfusion injury of the lungs of experimental animals.

(Thorax 1995;50:387-391)

Keywords: human thioredoxin, ischaemia reperfusion injury, lung, radical scavenger.

Ischaemia reperfusion injury has been studed widely in many transplantation and ischaemia models of animal organs including the heart, ${ }^{1}$ intestines, ${ }^{2}$ and kidney, ${ }^{3}$ and toxic oxygen metabolites have been shown to play a major part in this condition. In lung transplantation pulmonary capillary oedema occurs early after surgery and respiratory failure can occur; this is called "pulmonary reimplanting responses". These responses are considered to be due partly to disruption of nerves, lymphatic vessels, and bronchial vessels, as well as to ischaemic injury but, more importantly, to oxygen free radicals that are generated during reperfusion.

Human thioredoxin (hTRX) is a polypeptide consisting of 104 amino acids with a molecular weight of about $12000 .^{5}$ The active site of hTRX has the amino acid sequence -Cys-GlyPro-Cys- which participates in reduction reactions through reversible oxidation of its active centre dithiol to a disulphide. ${ }^{6}$ It is induced by a variety of stresses including $x$ irradiation, ultraviolet radiation, hydrogen peroxide $\left(\mathrm{H}_{2} \mathrm{O}_{2}\right)$, and mitogens. ${ }^{7}$ In isolated cells $\mathrm{hTRX}$ protects against the cytotoxicity of $\mathrm{H}_{2} \mathrm{O}_{2}$ as well as the cytotoxicity of tumour necrosing factor. ${ }^{89}$ hTRX has recently been reported to act as a scavenger of oxygen free radicals generated by xanthine oxidase. ${ }^{10}$

We have reported that in vivo rat lung models for ischaemia reperfusion injury are affected by environmental conditions, and have established a stable model for studying these effects by setting ischaemic time, temperature, and humidity. ${ }^{11}$ Using this model we have shown the protective effect of hTRX against ischaemia reperfusion injury. ${ }^{12}$ However, we did not perform any measurements except oxygenation and wet to dry ratios of lung weights.

Using this rat lung ischaemia reperfusion model we now present data which assess the protective effect of hTRX on ischaemia reperfusion injury of the lung.

\section{Methods}

Two kinds of experiments were designed. The first (Exp 1) was a time matched pair experiment in which treated and control animals were examined simultaneously. The second (Exp 2) was conducted under carefully controlled environmental conditions of temperature $25^{\circ} \mathrm{C}$ and humidity $65 \%$.

Male Wistar rats (body weight 240-260 g) were anaesthetised by subcutaneous injection of atropine sulphate $(0.01 \mathrm{mg})$ and intraperitoneal injection of pentobarbitone $(25 \mathrm{mg}$ / $\mathrm{kg}$ ) and pancuronium bromide $(0 \cdot 1 \mathrm{mg})$. The trachea was intubated with a 19 gauge intravenous cannula. In Exp 2 the trachea was ligated around the cannula so that air could not leak around the tube. Animals were mechanically ventilated with a Harvard pump using 
$100 \%$ oxygen, a tidal volume of $2.5 \mathrm{ml}$, a positive end expiratory pressure of $2 \mathrm{~cm} \mathrm{H}_{2} \mathrm{O}$, and a respiratory rate of 80 breaths/min. 24 gauge intravenous cannulae were inserted into the left carotid artery for sampling arterial blood and into the right femoral vein for the administration of phosphate buffered saline (PBS) or hTRX in PBS.

The sternum was cut transversely under a microscope (Zeiss Co Ltd, Oberkochen, Germany), extending to a bilateral thoracotomy at the fifth intercostal level, and the left pulmonary artery and left main bronchus were isolated. Heparin $(0 \cdot 1 \mathrm{ml})$ was injected into the left common carotid artery. The left pulmonary artery and the left main bronchus were clamped with microclips (Micro Clips, Edward Weck Co, Research Triangle Park, North Carolina, USA). In Exp 1 the bronchus was clamped at the end of expiration and in Exp 2 at the end of inspiration.

For Exp 1 the left lung was rendered ischaemic in the deflated state for 75 minutes. The clamps were then removed and the right pulmonary artery clamped. Operations were performed in matched pairs from the two groups (five hTRX treated rats, five controls). The same instruments were used and the entire procedure, including the operative manoeuvres, was carried out at an interval of 15 minutes in the treated and control animals. The room temperature was $25-27^{\circ} \mathrm{C}$ and the humidity $60-70 \%$.

In Exp 2, after the clamps had been removed, the right hilum was totally clamped under carefully controlled environmental conditions of $25^{\circ} \mathrm{C}$ and $65 \%$ humidity.

hTRX in PBS was injected into the right femoral vein of the treated rats immediately before reperfusion (hTRX $10 \mu \mathrm{g} / \mathrm{g}, 0.5 \mathrm{ml}$ ) and continuously for two minutes starting one minute after reperfusion (hTRX $20 \mu \mathrm{g} / \mathrm{g}, 1 \mathrm{ml}$ ). In the controls PBS was injected into the right femoral vein immediately before reperfusion $(0.5 \mathrm{ml})$ and for two minutes continuously starting one minute after reperfusion $(1 \mathrm{ml})$.

Plasmids possessing the hTRX gene were transformed in $E$ coli, multiplied by mass cultivation, and bacterial hTRX extracted. ${ }^{12} \mathrm{Re}-$ combinant hTRX was provided by Ajinomoto Company (Kawasaki, Japan).

The following measurements were also made:

Arterial blood gas analysis. Arterial blood $(0.2 \mathrm{ml})$ was drawn from the carotid artery 1,10 , and 20 minutes after reperfusion and analysed with an automated blood gas analyser (ABL300, Radiometer Company, Copenhagen, Denmark).

Wet/dry (W/D) ratio of lung tissue. The lower third of the left lung was weighed 20 minutes after reperfusion or at death (wet weight), and after being dried at $70^{\circ} \mathrm{C}$ for three days (dry weight), and the W/D ratio was calculated.

Histological examination (Exp 1 only). The upper third of the left lung was immersed in formalin 20 minutes after reperfusion (or at death). Tissue sections were stained with haematoxylin and eosin.

Biochemical assays (Exp 1 only). The middle third of the left lung was frozen immediately at $-20^{\circ} \mathrm{C} 20$ minutes after reperfusion (or at death). For the preparation of tissue extracts 10 volumes of ice cold $0.1 \mathrm{~mol}$ Tris- $\mathrm{Cl}, \mathrm{pH} \mathrm{7.5}$, 2 mmol EDTA was added to one volume frozen tissue. After homogenisation and centrifugation at $16000 \mathrm{rpm}$ for three minutes supernatants were collected and aliquots were stored at $-70^{\circ} \mathrm{C}$.

Assay for lipid peroxides (thiobarbituric acid reactants)..$^{13}$ The sample was mixed and heated at $95^{\circ} \mathrm{C}$ for 60 minutes and thiobarbituric acid reactants were extracted with $n$-butanol. The $\mathbb{\nabla}$ butanol phase was separated by centrifugation and the absorbance was measured at 532 and $553 \mathrm{~nm}$.

Glutathione peroxidase (GPx) assay. ${ }^{14}$ The $\overrightarrow{\vec{\omega}}$ assay mixture $(120 \mu \mathrm{l})$ contained $0.1 \mathrm{~mol}$ Tris$\mathrm{Cl}$, pH 7.5, $2 \mathrm{mmol}$ EDTA, $0.2 \mathrm{mmol} \underset{\vec{x}}{\vec{*}}$ NADPH, $1 \mathrm{mmol}$ sodium azide, $1 \mathrm{mmol}$ re- ir duced glutathione, $0 \cdot 25$ units $/ \mathrm{ml}$ glutathione reductase (type IV from bakers yeast, Sigma Chemical Co, St Louis, Missouri, USA), and the sample. The reaction was started by the $\mathrm{O}$ addition of $\mathrm{H}_{2} \mathrm{O}_{2}$ to $0.15 \mathrm{mmol}$ and the determination was based on the oxidation of $\mathrm{NADPH}$ at $340 \mathrm{~nm}$ at $25^{\circ} \mathrm{C}$.

Superoxide dismutase (SOD) assay. ${ }^{15}$ SOD activity was determined by the cytochrome C on reduction assay. The assay mixture $(600 \mu \mathrm{l})$ contained $0 \cdot 1 \mathrm{~mol}$ Tris-Cl, $\mathrm{pH} 7 \cdot 5,2 \mathrm{mmol}$ EDTA, $0.05 \mathrm{mmol}$ xanthine, $2 \mu \mathrm{g} / \mathrm{ml}$ xanthine oxidase, $6 \mu \mathrm{mol}$ cytochrome $\mathrm{C}$, and the sample. The reduction of cytochrome $\mathrm{C}$ was monitored $\triangle$ at $550 \mathrm{~nm}$. One unit of SOD was defined as $\overline{\bar{a}}$ the amount of enzyme which inhibits the rate of cytochrome $\mathrm{C}$ reduction by $50 \%$.

Two other groups $(n=5)$ of rats without ischaemia were selected to examine the influence of hTRX on these biochemical values. These animals were injected with PBS or PBS with $\mathrm{hTRX}(30 \mu \mathrm{g} / \mathrm{g})$ via the right femoral vein

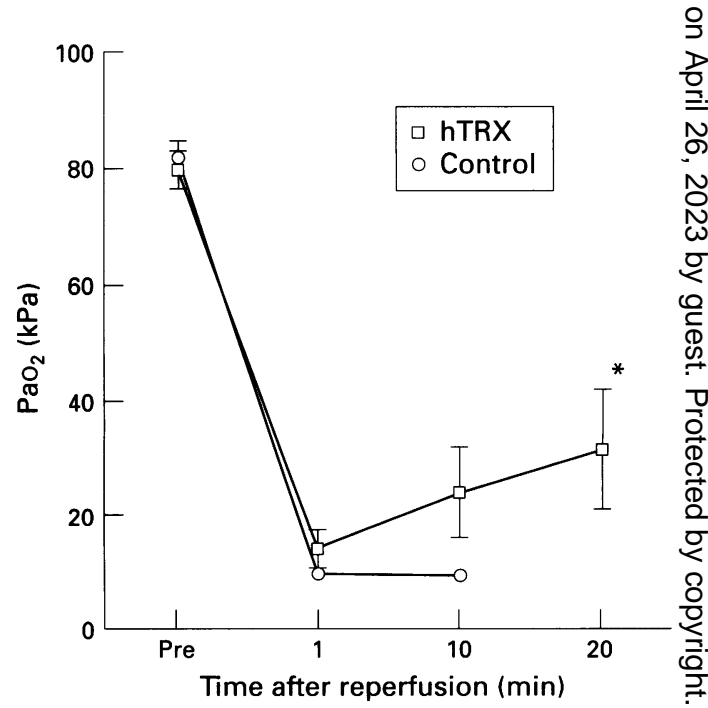

Figure 1 Mean (SE) $\mathrm{PaO}_{2}$ during reperfusion (Exp 1). One minute after the beginning of reperfusion $\mathrm{PaO}_{2}$ had fallen significantly from the preclamping level in both groups $(p<0 \cdot 05)$. In the group given human thioredoxin (hTRX) $\mathrm{PaO}_{2}$ was significantly higher 20 minutes after reperfusion compared with the level at one minute $(p<0 \cdot 05)$. 




Figure 2 Mean (SE) $\mathrm{PaO}_{2}$ during reperfusion (Exp 2). In the group given human thioredoxin (hTRX) $\mathrm{PaO}_{2}$ did not fall after reperfusion and was significantly higher than the control group 20 minutes after reperfusion $(p<0 \cdot 05)$.

and ventilated for 20 minutes without any ischaemia. The middle third of the left lung was examined as above.

All animals received humane care in compliance with the Care and Use of Laboratory Animals published by the National Institute of Health (NIH Publication No 85-23, revised in 1985).

\section{DATA ANALYSIS}

All values were expressed as mean (SE). Arterial blood gas values were statistically analysed by the analysis of variance (ANOVA). Paired Student's $t$ tests were used for W/D ratios and biochemical assays. A p value of less than 0.05 was considered to be significant.

\section{Results}

In Exp 1 all five animals in the control group died $3,5,10,18$, and 18 minutes after reperfusion (10.8 (3.2) minutes). In the hTRX group all five animals survived 20 minutes and the survival time was significantly different $(p<0.05)$ between the two groups. In Exp 2 one animal died one minute and one 10 minutes after reperfusion in the control group. In the hTRX group all four animals survived for 20 minutes.

In Exp 1 (fig 1) the $\mathrm{PaO}_{2}$ was $82 \cdot 0(2 \cdot 9) \mathrm{kPa}$ in the control group and $80.0(3.3) \mathrm{kPa}$ in the hTRX group before the clamping of the left pulmonary artery. One minute after reperfusion it was $9.9(1.4) \mathrm{kPa}$ and $14.3(3.4) \mathrm{kPa}$, respectively, a significant decrease in both groups $(\mathrm{p}<0.01)$. The $\mathrm{PaO}_{2}$ was $31.9(10.7) \mathrm{kPa}$ in the hTRX group 20 minutes after reperfusion, a significant increase $(p<0.05)$ above the value one minute after reperfusion, but it remained significantly less than the preclamping value $(\mathrm{p}<0.05)$.

In Exp 2 (fig 2) the $\mathrm{PaO}_{2}$ in the hTRX group did not fall after reperfusion, but in the control group it fell significantly 20 minutes after reperfusion. In the hTRX group the $\mathrm{PaO}_{2}$ was significantly higher than in the control group 20 minutes after reperfusion $(p<0.05)$.

The W/D ratio after Exp 1 was similar in the control group (6.64 $(0.65))$ and the hTRX group $(6 \cdot 23(0 \cdot 43))$. After Exp 2 the W/D ratio was also similar in the control $(6 \cdot 20(0 \cdot 78))$ and the hTRX groups $(6.28(0.43))$.

Histological examination with haematoxylin and eosin staining after Exp 1 showed thickening and mild oedema of the alveolar walls in all the control animals and in one of the five animals in the hTRX group. Alveolar exudation was noted in the two control animals that died 18 minutes after reperfusion (fig 3 ).

The level of lipid peroxides as thiobarbituric acid reactants (fig 4) in the control group in Exp 1 was $52 \cdot 1(7 \cdot 3) \mathrm{nmol} / \mathrm{g}$, significantly

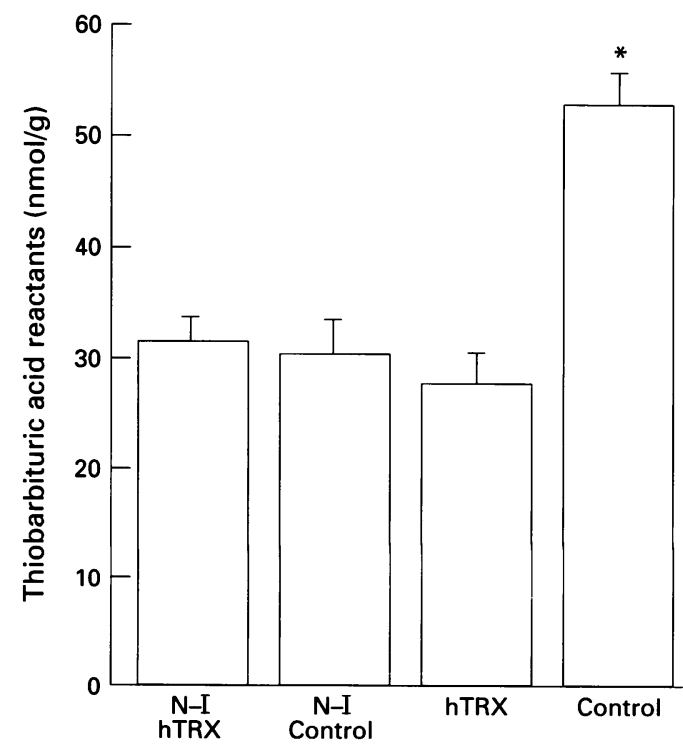

Figure 4 Assay for thiobarbituric acid reactants in the left lung. Values are mean (SE). N-I=non-ischaemic rats; $h T R X=$ human thioredoxin treated rats. ${ }^{*} p<0.05$ compared with each of the other three groups.
Figure 3 (A) Control group showing thickening and mild oedema of the alveolar walls with alveolar exudates. (B) hTRX group in which the alveolar structure is almost normal. Stain: haematoxylin and eosin, original magnification $\times 100$ reduced to $60 \%$ in origination.




Mean (SE) values of superoxide dismutase (SOD) and glutathione peroxidase (GPX)

\begin{tabular}{|c|c|c|c|c|}
\hline & \multicolumn{2}{|c|}{ Non-ischaemic groups } & \multicolumn{2}{|c|}{ Ischaemic groups } \\
\hline & $h T R X$ & Control & $h T R X$ & Control \\
\hline $\begin{array}{l}\text { SOD (units/g) } \\
\text { GPx (units/g) }\end{array}$ & $\begin{array}{l}473(37) \\
9 \cdot 7(0 \cdot 7)\end{array}$ & $\begin{array}{l}449(39) \\
9 \cdot 3(0 \cdot 8)\end{array}$ & $\begin{array}{l}427(23) \\
8 \cdot 0(0 \cdot 3)\end{array}$ & $\begin{array}{l}748(178)^{*} \\
13 \cdot 3(1 \cdot 3)^{*}\end{array}$ \\
\hline
\end{tabular}

${ }^{*} \mathrm{p}<0.05$ compared with the other three groups.

higher than in the hTRX group $(27 \cdot 7(2 \cdot 8)$ $\mathrm{nmol} / \mathrm{g}$ ) and the non-ischaemic groups. There was no difference between the two non-ischaemic groups.

The mean values of GPx and SOD activity in the control group were significantly higher than in the hTRX group (table; $\mathrm{p}<0.05$ ). There was no difference between the two non-ischaemic groups.

\section{Discussion}

Oxygen free radicals and oxygen metabolites generated during reperfusion are known to be involved in reperfusion injury. Active oxygen is produced by xanthine oxidase, neutrophils, and alveolar macrophages; however, the precise source of oxygen free radicals in ischaemia reperfusion injury remains to be clarified. ${ }^{16}$

Human thioredoxin contains the amino acid sequence -Cys-Gly-Pro-Cys- and closely resembles the thioredoxin of $E$ coli. ${ }^{17}$ It exists in either the reduced form (hTRX- $\left(\mathrm{SH}_{2}\right)$ ) with a dithiol, or the oxidised form (hTRX-S $)_{2}$. It participates in redox reactions through the reversible oxidation of its active centre dithiol to a disulphide and catalyses dithiol-disulphide exchange reactions. hTRX-S is generally reduced by NADPH and flavoprotein thioredoxin reductase. ${ }^{6}$ Spector et al reported that thioredoxin may significantly increase the ability of lens epithelial cells to recover from exposure to $\mathrm{H}_{2} \mathrm{O}_{2} .{ }^{8}$ Mitsui et al showed that hTRX could eliminate oxygen free radicals induced by xanthine oxidase. Since this reduction was suppressed by excess amounts of catalase but not by superoxide dismutase, they concluded that hTRX is a scavenger of hydrogen peroxide. ${ }^{10}$

In a previous study we established an in vivo model of ischaemia reperfusion injury in Wistar rats by clamping the left main bronchus and the left pulmonary artery for 75 minutes at a room temperature of $23^{\circ} \mathrm{C}$ and humidity of $65 \%$. We also reported that environmental conditions, including temperature and humidity, have a great influence on ischaemia reperfusion injury. ${ }^{11}$ Using this model we showed the protective effect of hTRX on rat lung reperfusion injury. However, we could not perform any assessments on the scavenger activity of hTRX. ${ }^{12}$ In the present study the protective effect against ischaemia reperfusion injury and the scavenger activity of hTRX were evaluated in this model of ischaemia reperfusion injury. Oxygen $(100 \%)$ was given as a higher oxygen concentration would increase ischaemia reperfusion injury. ${ }^{13}$

The rats perfused with $\mathrm{h} T R \mathrm{X}$ survived longer than the controls in both experiments. In Exp
$1 \mathrm{PaO}_{2}$ fell significantly and improved only in the hTRX group. Oxygenation is thought to be the best index for the evaluation of lung function ${ }^{18}$ and these results suggest that hTRX has a protective effect against ischaemia reperfusion injury of the lung.

A comparison of these two experiments shows that, in $\operatorname{Exp} 2, \mathrm{PaO}_{2}$ in the hTRX group did not fall as it did in Exp 1. This may be because (1) the ischaemia reperfusion injury in $\frac{\sigma}{\sigma}$ Exp 2 was not as severe as in Exp 1 since the left lung was not deflated during ischaemia; and (2) after reperfusion arteriovenous shunts in Exp 2 were less than in Exp 1 because the right hilum was totally clamped in Exp 2 preventing a decrease in the tidal volume.

Hydroxyl radicals can initiate a chain re- $\overrightarrow{\vec{\omega}}$ action of lipid peroxidation in endothelial cell $\stackrel{\sigma}{\omega}$ membranes. Lipid peroxides made thus con- $\overrightarrow{\vec{x}}$ tribute greatly to tissue damage, ultimately or leading to altered permeability and oedema. ${ }^{19}$ In this study lipid peroxide levels in the hTRX group were significantly lower than in the control group after reperfusion and the same in both the non-ischaemic groups, which suggests $\overrightarrow{-}$ that hTRX may act as a radical scavenger $\frac{D}{0}$ and suppress lung oedema. Our histological examination supported this hypothesis. How- $\vec{\bullet}$ ever, no significant difference was observed in 0 the $W / D$ ratios between the two groups. This may be because reperfusion injury is due, at least in part, to factors other than oxygen free radicals, or because there was insufficient time to develop pulmonary oedema in the control $\stackrel{\mathbb{Q}}{\Omega}$ groups.

SOD is an effective radical scavenger which protects cells from oxidation by catalysing the dismutation of superoxide anion to $\mathrm{H}_{2} \mathrm{O}_{2} \cdot{ }^{18}$ $\mathrm{GPx}$ is a non-specific peroxide scavenger which catalyses the oxidation of glutathione. ${ }^{19}$ In this study tissue concentrations of both these radical scavengers were significantly lower in the hTRX group. Furthermore, the values of SOD and GPx in the ischaemia reperfusion injury group were higher than those in the non-ischaemic groups. On the other hand, the $\mathrm{PaO}_{2}$ at 20 minutes in the hTRX group was significantly higher than in the control group, and lipid peroxides in the hTRX group were lower than of in the control group. Although it is uncertain $N$ that enzymes of this type can be induced in such a short time, it is possible that the low SOD and GPx concentrations in the hTRX 0 group were due to the inhibition of reperfusion injury by hTRX.

In summary, the administration of hTRX ${ }^{0}$ improved survival and oxygenation and inhibited the production of lipid peroxides generated by active oxygen species in this rat model of ischaemia reperfusion injury. These results suggest that human thioredoxin may be effect- 8 ive as a radical scavenger in the treatment of reperfusion injury of the lung.

1 Stewart JR, Blackwell WH, Crute SL, Loughlin V, Greenfield LJ, Hess ML. Inhibition of surgically induced ischfield $\mathrm{LJ}$, Hess $\mathrm{ML}$. Inhibition of surgically induced isch$\underset{f \text { Thorac Cardiovasc Surg 1983;83:262-72. }}{\text { emia/reperfusion injury by oxygen free radical }}$

2 Granger DN, Rutili G, McCord JM. Super oxide radicals in feline intestinal ischemia. Gastroenterology 1981;81:22-9.

3 Parks DA, Bulkley GB, Granger N. Role of oxygen free radicals in shock, ischemia, and organ preservation. Surgery 1983;94:428-32. 
4 Siegelman SS, Sinha SBP, Veith FJ. Pulmonary reimplantation response. Ann Surg 1973;177:30-6.

5 Teshigawara K, Maeda M, Nishino K, Nikaido T, Uchiyama $\mathrm{T}$, Tsudo $\mathrm{M}$, et al. Adult $\mathrm{T}$ leukemia cells produce a lymphokine that augments interleukin 2 receptor exlymphokine that augments interleukin
pression. $7 \mathrm{Mol}$ Cell Biol 1985;2:17-26.

pression. F Mol Cell Biol 1985;2:17-26.
6 Holmgren A. Thioredoxin. Ann Rev Biochem 1985;54:237Holm.

7 Tagaya Y, Wakasugi H, Masutani H, Nakamura H, Iwata $\mathrm{S}$, Mitsui A, et al. Role of ATL-derived factor (ADF) in the normal and abnormal cellular activation: involvement of dithiol related reduction. Mol Immunol 1990;27:127989.

8 Spector A, Yan GZ, Huang RRC, McDermott MJ, Gascoyne PRC, Pigiet V. The effect of $\mathrm{H}_{2} \mathrm{O}_{2}$ upon thioredoxinenriched lens epithelial cells. $¥$ Biol Chem 1988;263:498490.

9 Matsuda M, Masutani H, Nakamura H, Miyajima S, Yamauchi A, Yonehara S, et al. Protective activity of adult T cell leukemia-derived factor (ADF) against tumor necrosis factor-dependent cytotoxicity on U937 cells. F Immunol 1991;147:3837-44.

10 Mitsui A, Hirakawa T, Yodoi J. Reactive oxygen-reducing and protein-refolding activities of adult $T$ cell leukemiaderived factor(ADF)/human thioredoxin. Biochem Biophys Res Commun 1992;186:1220-6.

11 Ohno N, Yokomise H, Fukuse T, Hirata T, Hitomi S, Wada
H. Preparation of in-vivo rat lung model for ischemiareperfusion injury. Thorac Cardiovasc Surg 1993;41:304-7.

12 Yokomise H, Fukuse T, Hirata T, Ohkubo K, Go T, Muro

$\mathrm{K}$, et al. Effect of recombinant human adult $\mathrm{T}$ cell leukemia-derived factor on rat lung reperfusion injury. Respiration 1994;61:99-104.

13 Ohkawa H, Ohishi N, Yagi K. Assay for lipid peroxides in animal tissues by thiobarbituric acid reaction. Anal Bio chem 1979;95:351-8.

14 Flohe L, Gunzler WA. Assays of glutathione peroxidase Methods Enzymol 1984;105:114-21.

15 Flohe L, Otting F. Superoxide dismutase assays. Methods Enzymol 1984;105:93-104.

16 Deeb GM, Grum CM, Lynch MJ, Guynn TP, Gallagher KP, Ljungman AG, et al. Neutrophils are not necessary for induction of ischemia-reperfusion lung injury. $\mathfrak{f} \mathrm{Appl}$ for induction of ischemia-re

17 Tagaya Y, Okada M, Sugie K, Kasahara T, Kondo N, Hamuro $\mathrm{J}$, et al. ATL-derived factor (ADF), an IL-2 receptor/Tac inducer homologous to thioredoxin; possible involvement of dithiol-reduction in the IL-2 receptor induction. EMBO ₹ 1989;8:757-64

18 Detterbeck FC, Keagy BA, Paull DE, Wilcox BR. Oxygen free radical scavengers decrease reperfusion injury in the lung transplantation. Ann Thorac Surg 1990;50:204-10.

19 Weiss SJ. Oxygen, ischemia and inflammation. Acta Physiol Scand 1986;548(Suppl):9-37. 